\title{
Cross-cultural validation of the Cardiac Depression Scale in Iran
}

Leila Gholizadeh PhD, MSc, BSc ${ }^{1}$, Yenna Salamonson PhD, MA, BSc ${ }^{2}$, Patricia M. Davidson PhD, Med, BA, ${ }^{3}$ Kobra Parvan MSc, BSc ${ }^{4}$, Steven A. Frost MPH, BN ${ }^{5}$, Sungwon Chang MStat, MSc(Stat) ${ }^{6}$, David L. Hare PhD MBBS ${ }^{7}$

1. Lecturer, Faculty of Nursing, Midwifery \& Health, University of Technology, Sydney, Australia \& Tabriz University of Medical Sciences, Tabriz, Iran

2. Senior Lecturer, School of Nursing, University of Western Sydney, Sydney, Australia

3. Professor of Cardiovascular and Chronic Care, School of Nursing and Midwifery, Curtin University of Technology, Sydney Campus, Australia

4. Senior Lecturer, School of Nursing, Tabriz University of Medical Sciences, Tabriz, Iran

5. Lecturer, School of Nursing, University of Western Sydney, Sydney, Australia

6. School of Biomedical \& Health Sciences, University of Western Sydney, Sydney, Australia

7. Professor \& Senior Cardiologist, Austin Health, Heidelberg, Australia

Word count (exc. figures/tables): 4185

\section{Corresponding Author:}

Yenna Salamonson

Senior Lecturer

School of Nursing, College of Health \& Science

University of Western Sydney

Campbelltown Campus, Building 7

Locked Bag 1797

Penrith South. DC 1797

New South Wales, Australia

Phone: 0246203322

Fax: 0246253161

International: Replace 02 with 612 after your international access code

Email: y.salamonson@uws.edu.au 


\section{Abstract}

Background: The Cardiac Depression Scale (CDS) is a disease-specific instrument for measuring depression in cardiac patients. This study was designed to validate the CDS in an Iranian population.

Methods: Translation and back translation of the 26- Item CDS scale was performed using recommended procedures. The Iranian translation of the CDS (I-CDS) was administered to 261 individuals in Iran, concurrently with the Beck Depression Inventory. The factor structure of the I-CDS was examined using exploratory factor analysis procedures to enable comparison with previous psychometric evaluation of the CDS. Receiver operating characteristic (ROC) curves were used to examine the ability of the I-CDS to discriminate between categories of depression.

Results: First-order exploratory factor analysis uncovered two robust factors, consistent with the second-order dimensions originally reported by the developers of this instrument. Cronbach’s alpha was 0.88 for the total 26-item I-CDS, indicating satisfactory internal consistency of the I-CDS. Intercorrelation between the total scores for the I-CDS and BDI was $0.62(P<.001)$. For the I-CDS cut off of 90 , the sensitivity was $85 \%$, and specificity was 61\% with a computed area under the curve (AUC) of .81 (95\% CI, .76- .87). For the I-CDS cut off of 100 , the sensitivity was $81 \%$ and specificity was $63 \%$ with a computed AUC of .81 (95\% CI, .76- .87).

Conclusion: This validation study of the Iranian version of the CDS proved that it is an acceptable, a reliable and valid measure of depression in cardiac patients. 


\section{Introduction}

Depression, particularly mild depression, is common among those hospitalised with coronary heart disease (CHD) and has been estimated to affect about one-third of these individuals (Astin, Jones, \& Thompson, 2005; Lespérance \& Frasure-Smith, 2000). In one long-term follow-up study on mortality risk in people with heart disease, mild depression was shown to be associated with 38\% higher adjusted cardiac mortality risk (Barefoot et al., 1996). There is a dose response effect between untreated depression and health outcomes for people with CHD (Denollet, Vaes, \& Brutsaert, 2000). Of significance, individuals with CHD who are depressed are less likely to adhere to recommended lifestyle changes following an acute coronary event (Ziegelstein et al., 2000), and are at higher risk of recurrent cardiac events (Rozanski, Blumenthal, \& Kaplan, 1999). As a consequence, there is an increasing recognition of the importance of screening for depression in individuals with heart disease (Lichtman et al., 2008). The prevalence of depression in Iranian cardiac patients has not been reported, however, among the general population in Iran, the prevalence of minor depressive disorder, dysthymia and major depressive disorder was 5\%, 2.5\%, and 1\% respectively (Modabernia, Tehrani, Fallahi, Shirazi, \& Modabbernia, 2008).

Three large randomised trials have demonstrated that depression in people with heart disease can be improved by specific treatments (Berkman et al., 2003; Glassman et al., 2002; Lespérance et al., 2007). In spite of the targeted and conceptually congruent interventions, these studies have failed to demonstrate any reduction of cardiac events, particularly cardiac mortality (Berkman et al., 2003; Glassman et al., 2002; Lespérance et al., 2007). One explanation for these conflicting results may be the way depression was measured. A range of generic depression assessment tools was used with varying sensitivities in detecting depression in people with heart disease where cardiac-related symptoms, such as fatigue and 
lethargy, may emulate depressive symptoms. Hence, depression among individuals with heart disease is under-detected as somatic symptoms of depression are commonly attributed to cardiac problems instead of depression (Thornton, 2001).

Therefore, a disease-specific depression scale capable of accurately assessing depression among cardiac patients is a particularly useful assessment tool to ensure these patients receive appropriate management. The Cardiac Depression Scale (CDS), developed specifically to measure adjustment disorder with depressed mood among people with heart disease,(Hare \& Davis, 1996) has been shown to be more sensitive than generic depression scales such as the Beck Depression Inventory (BDI) in identifying depression, particularly in those with mild or subclinical depression $\{1$, \#6\}. The BDI, developed to detect, assess, and monitor changes in depressive symptoms among psychiatric patients, gives a skewed distribution on scores in people with heart disease (Hare \& Davis, 1996). The CDS and BDI both have excellent properties for detecting major depression in cardiac patients. However, the CDS is more accurate for detecting subtle changes and may be useful for monitoring changes across the illness trajectory (Frasure-Smith \& Lespérance, 2006). A CDS cut off score of $\geq 100$ provides $88 \%$ sensitivity and $84 \%$ specificity for detecting major depression. A cut off score of 90 provides $84 \%$ sensitivity and 78\% specificity for detecting minor depression (Wise, Harris, \& Carter, 2006).

The CDS has been validated on samples of ambulatory cardiac patients in Australia (Hare \& Davis, 1996; Wise et al., 2006), and replicated in the United Kingdom (Birks, Roebuck, \& Thompson, 2004). The cross-cultural robustness of the CDS has been demonstrated on a German version of this measure on a sample of participants recruited from an inpatient cardiac rehabilitation setting (Hare, Meyer, \& McBurney, 2000), and a Chinese version on a sample of inpatient cardiac patients (Wang, Thompson, Chairc, \& Hare, 2008). To date, factor 
analysis studies of the CDS have yielded slightly different findings. Although the original study uncovered 2 dimensions using second-order factor analysis (Hare \& Davis, 1996), both Birks et al. (2004) and Wise et al (2006) concluded that a one-factor solution was a better fit to their data using second-order factor analysis.

The purpose of this study was to develop an Iranian translation of the CDS (I-CDS) and to cross-validate this scale on Iranian inpatients with cardiovascular disease. In particular, the study aimed to explore the factor structure of the I-CDS, using exploratory factor analysis procedures to facilitate comparison with previous psychometric studies of the CDS. The reliability and the concurrent validity of the I-CDS were also examined, the latter by examining the relationship between I-CDS and the BDI.

\section{Methods}

\section{Study setting}

This study was conducted in the Province of Azerbaijan, north-west of Iran, where people mainly speak Azeri; however, majority of them can also speak, read and write in Farsi, the official language of the country.

\section{Sample}

The study participants were recruited from patients admitted to the intermediate cardiac care unit and cardiac surgical unit of a teaching heart hospital in Iran. Using non-random sampling method, 261 patients admitted to the intermediate cardiac care unit and cardiac surgical unit of a university-affiliated hospital were recruited from October 2007 to December 2007. The hospital is considered to be the biggest cardiac hospital in Tabriz and the north-west of Iran. Four patients refused to participate in the study mainly due to poor health. All participants had a diagnosis of heart disease including myocardial infarction (25\%), unstable angina (38\%), heart failure (17\%), angioplasty and/or cardiac surgery (20\%), as documented by a 
cardiologist in their medical records. This study received ethics clearance from both the university and the hospital where the study was undertaken.

\section{Materials}

\section{Beck Depression Inventory}

The Beck Depression Inventory (BDI) is a 21-question multiple choice self-report inventory, measuring the symptoms of depression using scores from 0 to 3 . The BDI is perhaps one of the most commonly used screening tools for depression, and has been widely used on a variety of populations with diverse diseases including heart disease. The BDI is a reliable and valid scale, with internal consistency of .86 for psychiatric patients and .81 for nonpsychiatric populations (Beck, Steer, \& Carbin, 1988a). The advantage of the BDI is the possibility of comparing depression scores in diverse populations and diseases (Beck et al., 1988a). The BDI has previously been validated in Iranian populations, with high internal consistency (Cronbach's alpha $=.87)$ and acceptable test-retest reliability $(r=.74)$ (Ghassemzadeh, Mojtabai, Karamghadiri, \& Ebrahimkhani, 2005).

\section{Cardiac Depression Scale}

The CDS is a 26-item self-rating scale, measuring depression specifically in individuals with diagnosed heart disease using a Likert scale of 1 to 7 . The CDS was originally developed in the English language and was validated in an Australian population, with a reliability coefficient of .9 and correlation with the BDI of .73 (Hare \& Davis, 1996). The original CDS was reported to yield two dimensions and seven subscales including sleep, anhedonia, uncertainty, mood, cognition, hopelessness and inactivity (Hare \& Davis, 1996).

\section{Translation and back-translation of the I-CDS}

Translation and back-translation of the I-CDS were conducted following the guidelines suggested by Guillemin and colleagues (Guillemin, Bombardier, \& Beaton, 1993). The 26 
items of the CDS were translated into Farsi by two clinicians with experience in the translation of English texts. The back-translation was performed by a bilingual expert who had not seen the scale before. The final Farsi version of the CDS was achieved following repeated translation and back-translation procedures until all bilingual experts in the research team confirmed equivalence of the English and Farsi versions. In line with the original CDS version, the final 26-item I-CDS contains 7 reverse-scored items.

\section{Procedure}

A research assistant trained in instrument administration explained the study in the participants’ first language and obtained verbal consent from the participants. A verbal consent was obtained from both participants and parents if the participant was under 18 years old. Participants were then asked to complete the I-CDS and the BDI independently. The questionnaires were collected a few days later. In instances where a participant was unable to read, the research assistant read the questionnaire out loud to the participant to obtain their responses.

\section{Data analysis}

To determine the factor structure of the I-CDS, exploratory factor analyses were used. Although confirmatory factor analysis is recommended for validated scales such as the CDS (Henson \& Roberts, 2006), the conflicting findings on the factor structures warranted further exploratory validation of this instrument. Hence, we conducted first-order exploratory factor analysis to examine the factor structure of the I-CDS using previously reported extraction procedures (Birks et al., 2004; Hare \& Davis, 1996; Wise et al., 2006). A factor loading of .3 or greater, indicating a shared variance with the factor of at least $10 \%$ was used as the cut off for significant loading. Internal consistency determined by Cronbach’s alpha was used to assess the reliability of I-CDS. Receiver-operating characteristic (ROC) curve was used as a graphical tool for assessing the capability of I-CDS as a screening tool to discriminate 
between those with or without depressive symptoms. Sensitivity, specificity and area under ROC curve were performed using the BDI as the comparison measure. Total I-CDS and the BDI scores with recommended cut off scores of 90 for I-CDS and 10 for BDI for mild depression, and 100 for I-CDS and 18 for BDI for severe depressive symptoms were used (Beck, Steer, \& Garbin, 1988b; Grace et al., 2005; Wise et al., 2006).

\section{Results}

Of the 261 participants completing the survey, about two-thirds (67.8\%) were males and 82.6\% married. The mean age was 52 ( $\mathrm{SD}=13.8$ years; range $14-84$ ) and approximately $90 \%$ of the participants had at least primary schooling. Nearly all of the participants self-declared their religion to be Muslims (96.2\%), and 40\% had a previous diagnosis of heart disease.

The mean total I-CDS score was 101.37 (SD=25.72; median 102; range 40-173), with skewness of -.09 and kurtosis of -.18, and it fulfilled the Kolmogorov-Smirnov test of normal distribution $(\mathrm{p}=.54)$. In comparison, the mean total BDI score was 15.61 (SD=10.77; median 13; range 0-57), with skewness of .91 and kurtosis of .53, and it failed the KolmogorovSmirnov test of normal distribution $(\mathrm{p}<.001)$.

\section{Factor structures}

In order to reproduce the CDS factor structure reported previously, we replicated the factor analysis procedures used by Wise et al.(Wise et al., 2006), Birks et al.(Birks et al., 2004) and Hare et al.(Hare \& Davis, 1996) Using the ‘eigenvalues of greater than one’ criterion for factor extraction, seven factors were extracted that accounted for $61 \%$ of variance. Using the maximum likelihood factor extraction with oblique rotation as reported in the original validation (Hare \& Davis, 1996), items of all subscales had factor loadings above the .3 threshold, but cross factor loadings were detected in 3 of the 7 subscales, which were mood, anhedonia and uncertainty. 
Using Catell's scree plot analysis to determine the number of factors to retain, six factors were extracted accounting for $57 \%$ of the variance, which is consistent with the number of factors reported by Wise et al.(Wise et al., 2006). Applying the same procedure as Wise et al.(Wise et al., 2006) of principal components with orthogonal (varimax) rotation, there were shared variance and cross-loadings of items, particularly in 2 subscales, fear and mood (Table 1).

In keeping with the procedure reported by Birks et al.(Birks et al., 2004) a one-factor solution using principal axis factoring extraction uncovered 24 items with a factor loading that were above the .3 cut off. Two items that failed to reach salient factor loading (i.e. less than .3) were: Item 15 'My mind is as fast and alert as always' and item 2 'My concentration is as good as it ever was'.

The authors of the scale suggest that the CDS consists of two underlying dimensions and 7 subscales. A first-order two-factor extraction, using the maximum likelihood factor extraction with oblique rotation yielded the same two structure of significant factor loadings ( .30 to .70) similar to the two dimensions previously reported in the original validation (Hare \& Davis, 1996). The first factor (dimension A) consists of items from Hare \& Davis (Hare \& Davis, 1996) original 5 subscales (sleep, uncertainty, mood, hopelessness and inactivity), and the second factor (dimension B) consists of a total of 7 items from the original remaining 2 subscales: anhedonia and cognition (Table 2).

Cronbach’s alpha was .88 for the total 26-item I-CDS, indicating satisfactory internal consistency of the I-CDS. Cronbach's alpha for each of the factors were .88 for dimension A and .70 for dimension B. Age and scores of the dimension A $(r=.14, P=.029)$ and dimension $\mathrm{B}(r=.15, P=.023)$ correlated weakly, demonstrating that these scores to a great extent are 
independent of age with is consistent with the report of the original study (Hare \& Davis, 1996).

\section{Comparisons between I-CDS and BDI}

Intercorrelation between the total scores for the I-CDS and BDI was .62 $(P<.001)$. Using the recommended BDI cut off score of 10 for mild to moderate depression and 18 for severe depression, two receiver operating characteristic (ROC) curves were used to examine the ability of the I-CDS to discriminate between the two categories of depression. For the I-CDS cut off of 90 , the sensitivity was $85 \%$, and specificity was $61 \%$ with a computed area under the curve (AUC) of .81 (95\% CI, .76- .87) when discriminating mild and moderate depression from individuals without depressive symptoms. For the I-CDS cut off of 100, the sensitivity was $81 \%$, and specificity was $63 \%$ with a computed AUC of .81 (95\% CI, .76- .87) (Table 3, Figure 1). The percentages of patients with mild/moderate and severe depression according to the I-CDS and BDI are presented in Table 4.

\section{Discussion}

The findings presented above provided validation of the I-CDS and the suitability of the measure in the Iranian cardiac population. Compared to the previous studies, the higher ICDS scores and BDI scores may be partially explained by differences in study settings.

Achieving normative data in particular populations is important and may need to be considered within a context of acculturation and cultural competence (Davidson et al., 2007). These observations raise an important question as to the potential influence of culture and socialisation in the expression of depressive symptoms (Kleinman, 2004) and cultural tendencies in response styles (Hamamura, Heine, \& Paulhus, 2008), and therefore requires further investigation and particularly consideration in clinical practice. This is not just important for validating psychometric tools in specific countries but also in assessing for 
depression in countries with large culturally and linguistically diverse populations, such as Australia, the United Kingdom and the United States of America. This is likely to become of increasing importance as emphasis is placed on the influence of depression on the illness trajectory. Therefore, further cross-validation studies are needed to test the CDS against a clinical interview by an experienced/trained health expert. Appreciating the cultural dimension may also be important in determining the individual's attribution of causality and treatment options. This may influence interpersonal relationships and the interaction with treating clinicians.

The results of this study showed I-CDS to be moderately correlated with the BDI, with a similar value to the correlation values reported by Hare and Davis (Hare \& Davis, 1996), Birks et al. (Birks et al., 2004) and Di Benedetto et al. $\{1, \# 6\}$. In addition, consistent with findings from the previous studies (Birks et al., 2004; Hare \& Davis, 1996; Wise et al., 2006), the I-CDS showed a normal distribution of scores indicating the greater sensitivity of the scale to both extremes of scores compared to the BDI scores, which makes the I-CDS as a useful scale to measure depression in cardiac patients who are more likely to suffer from mild depression which may have an adverse impact on their recovery from a cardiac event (Astin et al., 2005; Irvine et al., 1999). Although slightly lower than those reported in the previous studies (Birks et al., 2004; Hare \& Davis, 1996; Hare et al., 2000; Wise et al., 2006), this study demonstrated acceptable internal consistency for the overall I-CDS (Hare \& Davis, 1996). The results of factor analysis using the same extraction procedure as reported by previous studies suggest that the factor structure of I-CDS supports the findings of the original study, further supporting the two-dimension structure of the CDS, labelled as A and B by Hare and Davis (Hare \& Davis, 1996). 
This study has shown that the CDS is a valid tool for assessing depression in cardiac patients. The CDS can also facilities studying subtle trends in depression in cardiac patients and monitoring depression over time (Frasure-Smith \& Lespérance, 2006). Effective management of depression has a potential to help patients cope better with their disease and comply with medical recommendations (Ziegelstein et al., 2000). This underscores the importance of screening for and treating depression in the acute care setting. The usefulness of the CDS for outcome measurement such as response to treatment or effectiveness of interventions such as cardiac rehabilitation programs should be addressed by further studies.

Further, the answer of timing of assessment and initiation of management of depressive symptoms in heart disease remains elusive and further investigation is warranted given the burden of this problem. Participants were recruited from an acute care setting in which cardiac patients are more likely to experience "adjustment disorder with depressed mood”. For example, in a study about half of patients had major or minor depression one week after experiencing myocardial infarction and one third met the criteria 3 to 4 months later

(Schleifer et al., 1989). It is also likely that depression may have preceded the development of heart disease in some participants.

\section{Conclusion}

Depression is increasingly recognised as influencing outcomes in individuals with heart disease. The study has shown that the I-CDS is a sensitive, reliable and valid disease-specific scale to screen for depression. Further cross-validation studies are advised to test I-CDS against clinical rating of depression. 


\section{References}

Astin, F., Jones, K., \& Thompson, D. R. (2005). Prevalence and patterns of anxiety and depression in patients undergoing elective percutaneous transluminal coronary angioplasty. Heart and Lung, 34(6), 393-401.

Barefoot, J. C., Helms, M. J., Mark, D. B., Blumenthal, J. A., Califf, R. M., Haney, T. L., et al. (1996). Depression and long-term mortality risk in patients with coronary artery disease. American Journal of Cardiology, 78(6), 613-617.

Beck, A. T., Steer, R. A., \& Carbin, M. G. (1988a). Psychometric properties of the Beck Depression Inventory: Twenty-five years of evaluation. Clinical Psychology Review, 8(1), 77-100.

Beck, A. T., Steer, R. A., \& Garbin, M. G. (1988b). Psychometric properties of the Beck Depression Inventory: twenty-five years of evaluation. Clinical Psychology Review, 8(1), 77-100.

Berkman, L. F., Blumenthal, J., Burg, M., Carney, R. M., Catellier, D., Cowan, M. J., et al. (2003). Effects of treating depression and low perceived social support on clinical events after myocardial infarction: the enhancing recovery in coronary heart disease patients (ENRICHD) randomized trial. Journal of the American Medical Association, 289(23), 3106-3116.

Birks, Y., Roebuck, A., \& Thompson, D. R. (2004). A validation study of the Cardiac Depression Scale (CDS) in a UK population. British Journal of Health Psychology, 9(Part 1), 15-24.

Davidson, P. M., MacDonald, P., Moser, D. K., Ang, E., Paull, G., Choucair, S., et al. (2007). Cultural diversity in heart failure management: Findings from the DISCOVER study (Part 2). Contemporary Nurse, 25(1-2), 50-62.

Denollet, J., Vaes, J., \& Brutsaert, D. L. (2000). Inadequate response to treatment in coronary heart disease: Adverse effects of type D personality and younger age on 5-year prognosis and quality of life. Circulation 102, 630-635. 
Di Benedetto, M., Lindner, H., Hare, D. L., \& Kent, S. (2006). Depression following acute coronary syndromes: A comparison between the Cardiac Depression Scale and the Beck Depression Inventory II. Journal of Psychosomatic Research, 60(1), 13-20.

Frasure-Smith, N., \& Lespérance, F. (2006). Recent evidence linking coronary heart disease and depression. Canadian Journal of Psychiatry, 51(12), 730-737.

Ghassemzadeh, H., Mojtabai, R., Karamghadiri, N., \& Ebrahimkhani, N. (2005). Psychometric properties of a Persian-language version of the Beck Depression Inventory - Second edition: BDI-II PERSIAN. Depression and anxiety, 21, 185-192.

Glassman, A. H., O'Connor, C. M., Califf, R. M., Swedberg, K., Schwartz, P., Bigger Jr, J. T., et al. (2002). Sertraline treatment of major depression in patients with acute MI or unstable angina. Journal of the American Medical Association, 288(6), 701709.

Grace, S. L., Abbey, S. E., Kapral, M. K., Fang, J., Nolan, R. P., \& Stewart, D. E. (2005). Effect of depression on five-year mortality after an acute coronary syndrome. American Journal of Cardiology, 96(9), 1179-1185.

Guillemin, F., Bombardier, C., \& Beaton, D. (1993). Cross-cultural adaptation of healthrelated quality of life measures: Literature review and proposed guidelines. Journal of Clinical Epidemiology, 46(12), 1417-1432.

Hamamura, T., Heine, S. J., \& Paulhus, D. L. (2008). Cultural differences in response styles: The role of dialectical thinking. Personality and Individual Differences, 44(4), 932-942.

Hare, D. L., \& Davis, C. R. (1996). Cardiac Depression Scale: Validation of a new depression scale for cardiac patients. Journal of Psychosomatic Research, 40(4), 379-386.

Hare, D. L., Meyer, K., \& McBurney, H. (2000). Cross-cultural robustness of depressed mood in cardiac patients -- The German language version of the cardiac depression scale. Heart, Lung and Circulation, 9(3), A167. 
Henson, R. K., \& Roberts, J. K. (2006). Use of exploratory factor analysis in published research: common errors and some comment on improved practice. Educational and Psychological Measurement, 66(3), 393-416.

Irvine, J., Basinski, A., Baker, B., Jandciu, S., Paquette, M., Cairns, J., et al. (1999).

Depression and risk of sudden cardiac death after acute myocardial infarction: Testing for the confounding effects of fatigue. Psychosomatic Medicine, 61(6), 729.

Kleinman, A. (2004). Culture and depression. New England Journal of Medicine, 351(10), 951-953.

Lespérance, F., \& Frasure-Smith, N. (2000). Depression in patients with cardiac disease: A practical review. Journal of Psychosomatic Research, 48(4-5), 379-391.

Lespérance, F., Frasure-Smith, N., Koszycki, D., Laliberte, M. A., Van Zyl, L. T., Baker, B., et al. (2007). Effects of citalopram and interpersonal psychotherapy on depression in patients with coronary artery disease: The Canadian Cardiac Randomized Evaluation of Antidepressant and Psychotherapy Efficacy (CREATE) trial. Journal of the American Medical Association, 297(4), 367379.

Lichtman, J. H., Bigger, J., JT., Blumenthal, J. A., Frasure-Smith, N., Kaufmann, P. G., \& Lesperance, F., et al. (2008). Depression and coronary heart disease: Recommendations for screening, referral, and treatment. A Science Advisory From the American Heart Association prevention Committee of the Council on Cardiovascular Nursing, Council on Clinical Cardiology, Council on Epidemiology and Prevention, and Interdisciplinary Council on Quality of Care and Outcomes Research: Endorsed by the American Psychiatric Association. Circulation, 118(17), 1768-1775.

Modabernia, M., Tehrani, H., Fallahi, M., Shirazi, M., \& Modabbernia, A. (2008). Prevalence of depressive disorders in Rasht, Iran: A community based study. Clinical Practice and Epidemiology in Mental Health, 4(1), 20. 
Rozanski, A., Blumenthal, J. A., \& Kaplan, J. (1999). Impact of psychological factors on the pathogenesis of cardiovascular disease and implications for therapy. Circulation, 99(16), 2192-2217.

Schleifer, S. J., Macari-Hinson, M. M., Coyle, D. A., Slater, W. R., Kahn, M., Gorlin, R., et al. (1989). The nature and course of depression following myocardial infarction. Archives of Internal Medicine, 149(8), 1785-1789.

Thornton, L. A. (2001). Depression in post-acute myocardial infarction patients. Journal of the American Academy of Nurse Practitioners, 13(8), 364-367.

Wang, W., Thompson, D. R., Chairc, S. Y., \& Hare, D. L. (2008). A psychometric evaluation of a Chinese version of the Cardiac Depression Scale. Journal of Psychosomatic Research 65, 123-129.

Wise, F. M., Harris, D. W., \& Carter, L. M. (2006). Validation of the Cardiac Depression Scale in a cardiac rehabilitation population. Journal of Psychosomatic Research, 60(2), 177-183.

Ziegelstein, R. C., Fauerbach, J. A., Stevens, S. S., Romanelli, J., Richter, D. P., \& Bush, D. E. (2000). Patients with depression are less likely to follow recommendations to reduce cardiac risk during recovery from a myocardial infarction. Archives of Internal Medicine, 160(12), 1818-1823. 
Table 1

Factor loadings: A six-factor extraction of the I-CDS (Principal Components with Varimax rotation)

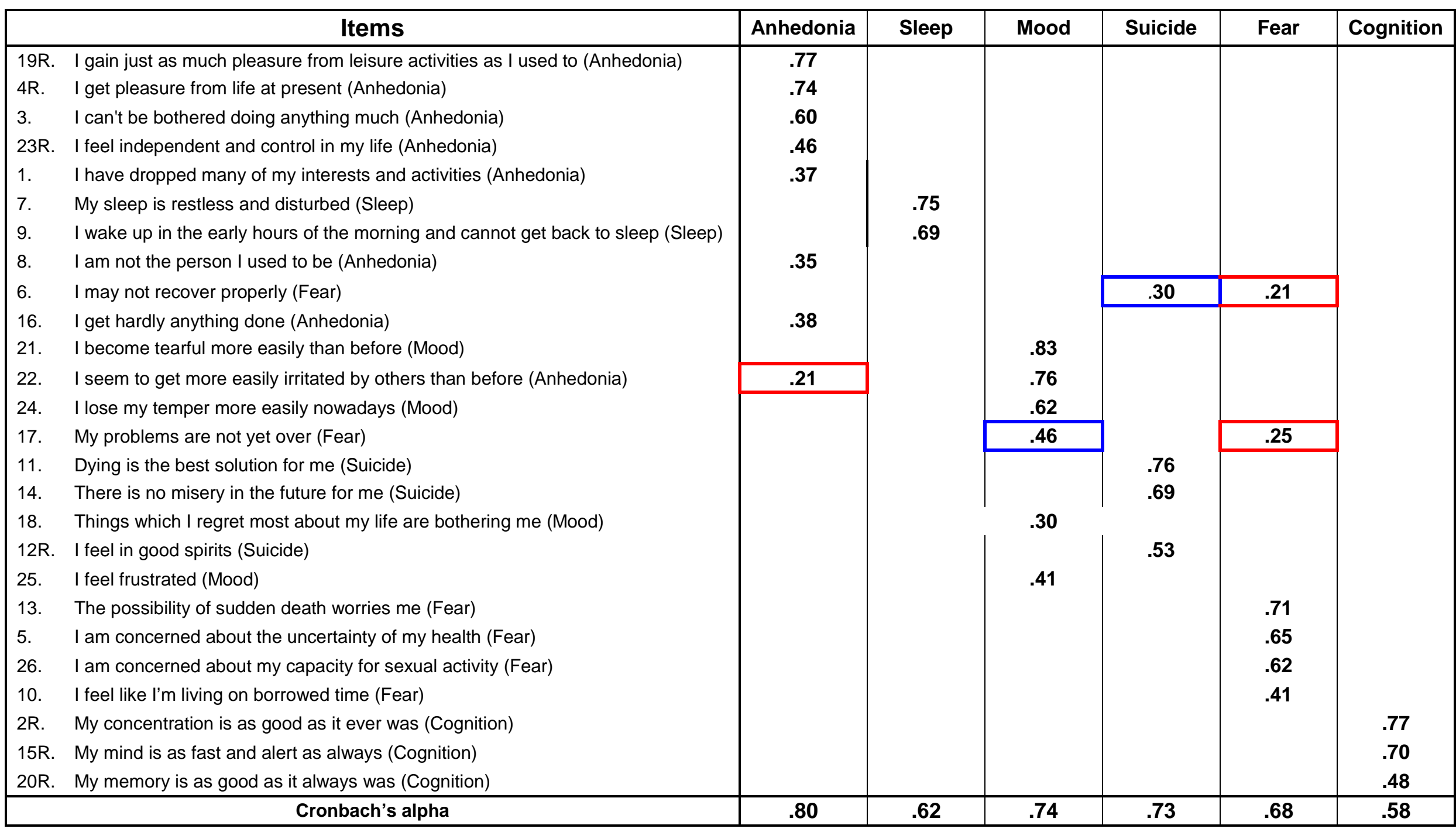

Factor loading $<.30$

Cross-loading on other subscales 


\begin{tabular}{|c|c|c|c|}
\hline & & \multicolumn{2}{|c|}{ Factor Loadings } \\
\hline & & $\begin{array}{c}\text { Factor A: } \\
\text { Mood, Inactivity, } \\
\text { Sleep, } \\
\text { Hopelessness, } \\
\text { Uncertainty } \\
\end{array}$ & $\begin{array}{l}\text { Factor B: } \\
\text { Anhedonia } \\
\text {, Cognition }\end{array}$ \\
\hline 22. & I seem to get more easily irritated by others than before (Mood) & .70 & \\
\hline 21. & I become tearful more easily than before (Mood) & .67 & \\
\hline 25. & I feel frustrated (Mood) & .65 & \\
\hline 16. & I get hardly anything done (Inactivity) & .63 & \\
\hline 14. & There is no misery in the future for me (Hopelessness) & .61 & \\
\hline 17. & My problems are not yet over (Uncertainty) & .60 & \\
\hline 8. & I am not the person I used to be (Uncertainty) & .56 & \\
\hline 1. & I have dropped many of my interests and activities (Inactivity) & .56 & \\
\hline 7. & My sleep is restless and disturbed (Sleep) & .54 & \\
\hline 6. & I may not recover properly (Uncertainty) & .53 & \\
\hline 24. & I lose my temper more easily nowadays (Mood) & .52 & \\
\hline 10. & I feel like I'm living on borrowed time (Hopelessness) & .44 & \\
\hline 5. & I am concerned about the uncertainty of my health (Uncertainty) & .43 & \\
\hline 13. & The possibility of sudden death worries me (Uncertainty) & .42 & \\
\hline 9. & $\begin{array}{l}\text { I wake up in the early hours of the morning and cannot get back to sleep } \\
\text { (Sleep) }\end{array}$ & .41 & \\
\hline 3. & I can't be bothered doing anything much (Inactivity) & .40 & \\
\hline 11. & Dying is the best solution for me (Hopelessness) & .36 & \\
\hline 26. & I am concerned about my capacity for sexual activity (Mood) & .35 & \\
\hline 18. & Things which I regret most about my life are bothering me (Uncertainty) & .30 & \\
\hline $20 \mathrm{R}$. & My memory is as good as it always was (Cognition) & & .62 \\
\hline $12 \mathrm{R}$. & I feel in good spirits (Anhedonia) & & .57 \\
\hline $15 \mathrm{R}$. & My mind is as fast and alert as always (Cognition) & & .44 \\
\hline 19R. & I gain just as much pleasure from leisure activities as I used to (Anhedonia) & & .43 \\
\hline 4R. & I get pleasure from life at present (Anhedonia) & & .42 \\
\hline & My concentration is as good as it ever was (Cognition) & & .34 \\
\hline 23R. & I feel independent and control in my life (Cognition) & & .32 \\
\hline
\end{tabular}


Table 2 Sensitivity and specificity comparison of I-CDS with BDI (n =261)

\begin{tabular}{|c|c|c|c|c|}
\hline \multirow{2}{*}{$\begin{array}{c}\text { Total I-CDS } \\
\text { score }\end{array}$} & \multicolumn{2}{|c|}{ BDI cutoff: >10 } & \multicolumn{2}{|c|}{ BDI cutoff: $>18$} \\
\hline & Sensitivity & 1 - Specificity & Sensitivity & 1 - Specificity \\
\hline 39.0 & 1.00 & 1.00 & 1.00 & 1.00 \\
\hline 49.0 & .99 & .95 & 1.00 & .97 \\
\hline 60.0 & .99 & .79 & 1.00 & .88 \\
\hline 70.5 & .97 & .67 & 1.00 & .79 \\
\hline 80.5 & .93 & .56 & .99 & .70 \\
\hline 90.5 & .85 & .39 & .94 & .55 \\
\hline 100.5 & .68 & .25 & .81 & .37 \\
\hline 110.5 & .51 & .08 & .66 & .20 \\
\hline 120.5 & .32 & .05 & .46 & .09 \\
\hline 131.0 & .20 & .01 & .31 & .04 \\
\hline 140.5 & .07 & .00 & .12 & .01 \\
\hline 151.0 & .04 & .00 & .08 & .00 \\
\hline 161.0 & .01 & .00 & .01 & .00 \\
\hline
\end{tabular}




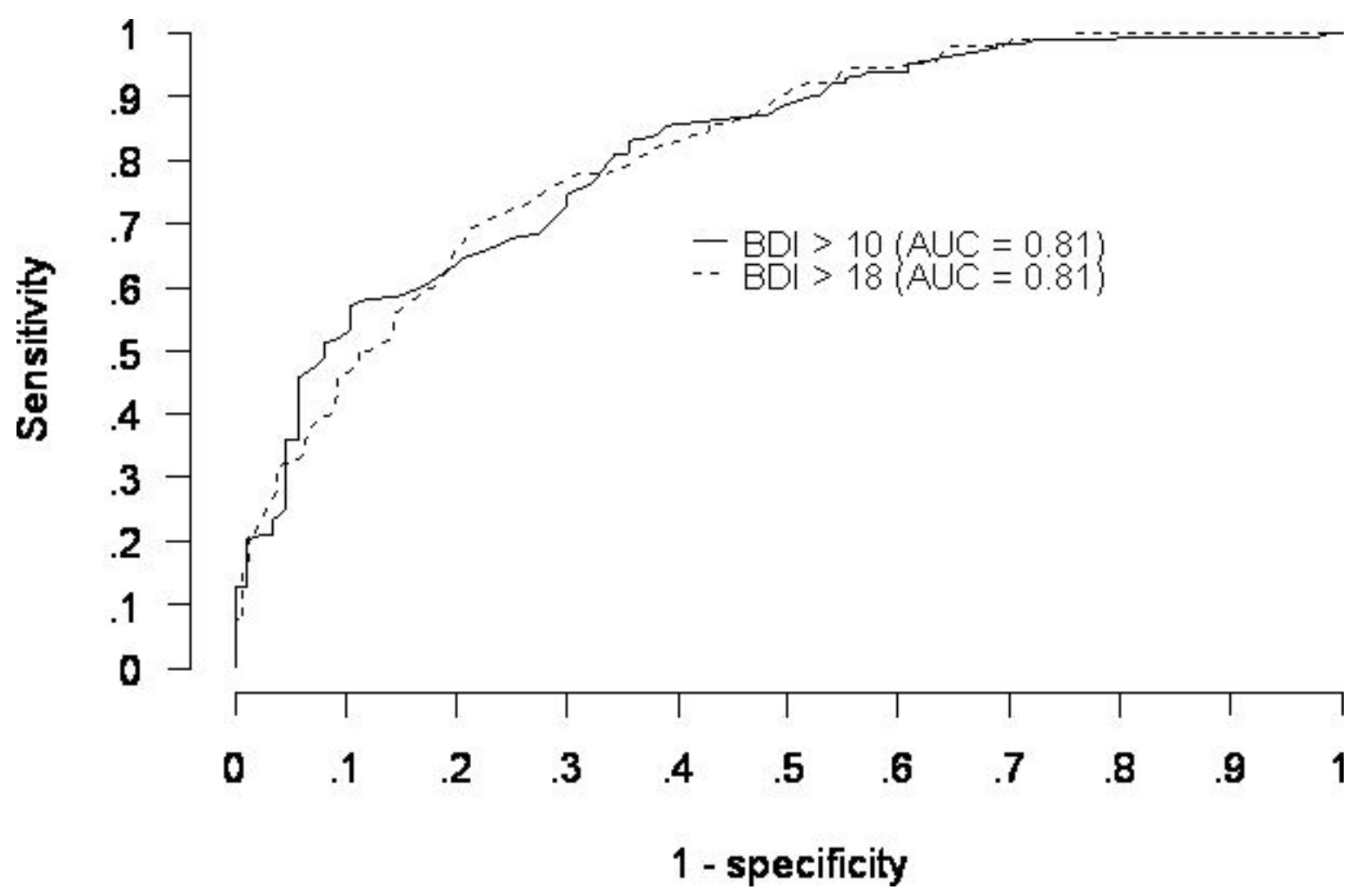

Figure 1 ROC curves for detection by CDS for mild to moderate (BDI > 10) and severe depression (BDI > 18) 
Table 4 The percentages of patients with mild/moderate and severe depression according to the I-CDS and BDI

\begin{tabular}{|c|c|c|c|c|c|}
\hline \multicolumn{2}{|c|}{ Not depressed } & \multicolumn{2}{c|}{ Mild/Moderate depression } & \multicolumn{2}{c|}{ Severe depression } \\
\hline I-CDS $\leq 90$ & BDI $\leq 10$ & $90<$ I-CDS $<100$ & $10<$ BDI $<18$ & I-CDS $\geq 100$ & BDI $\geq 18$ \\
\hline $30 \%$ & $38 \%$ & $17 \%$ & $26 \%$ & $53 \%$ & $36 \%$ \\
\hline
\end{tabular}

